\title{
Adsorção de dióxido de carbono utilizando o biochar produzido a partir de sementes de mangaba (Hancornia speciosa)
}

\author{
Adsorption of carbon dioxide using a biochar produced from mangaba seeds (Hancornia \\ speciosa) \\ L. F. S. Moura*; J. J. Marques; J. A. Freitas \\ Departamento de Engenharia Ambiental/Laboratório de Controle e Poluição do Ar, Universidade Federal de \\ Sergipe, 49100-000, São Cristóvão-Sergipe, Brasil.
}

*layfernandes20@gmail.com

(Recebido em 31 de janeiro de 2018; aceito em 12 de março de 2018)

\begin{abstract}
A concentracão dos gases de efeito estufa tem aumentado consideravelmente na atmosfera terrestre devido às acões antrópicas, intensificando o aquecimento global e desencadeando mudancas climáticas. Por conta desse cenário, acões estão sendo tomadas para reduzir as emissões desses poluentes, sobretudo o dióxido de carbono. CO,. O Protocolo de Kvoto (Kvoto. 1997) e a $21^{a}$ Conferência das Partes. COP21 (Paris. 2015). são exemplos de acordos globais para a reducão das mudancas climáticas. Nesse contexto, tecnologias avancadas para o sequestro de dióxido de carbono estão em desenvolvimento, dentre as quais aquelas baseadas na adsorcão física. $\mathrm{O}$ estudo integra essa classe de processos e utilizou um biochar produzido através da pirólise das sementes da mangaba (Hancornia speciosa) como adsorvente. O biochar foi caracterizado quanto à área superficial, diâmetro de poros, morfologia e determinacão dos grupos funcionais. A adsorcão de $\mathrm{CO}_{2}$ foi realizada utilizando-se a técnica volumétrica estática. variando-se a pressão inicial do sistema que consistiu numa coluna de leito fixo em escala semi-piloto. Os dados experimentais foram aiustados a modelos da literatura. O modelo de Freundlich foi o que melhor se aiustou, constatando a adsorcão em multicamadas. A cinética de adsorcão seguiu o modelo de difusão intrabartícula. indicando que a difusão é a etapa limitante do processo. O valor máximo de capacidade adsortiva obtido para o biochar foi $5,21 \mathrm{~mol} \mathrm{CO}_{2} / \mathrm{kg}$ de bioadsorvente.

Palavras-chave: Aquecimento global; adsorção; biochar.
\end{abstract}

The concentration of greenhouse gases has increased considerably in the Earth's atmosphere due to human actions, intensifying global warming and triggering climate change. Because of this scenario, actions are being taken to reduce emissions of these pollutants, especially carbon dioxide, $\mathrm{CO}_{2}$. The Kyoto Protocol (Kyoto, 1997) and the 21st Conference of the Parties, COP21 (Paris, 2015), are examples of global agreements to reduce climate change. In this context, advanced technologies for the sequestration of carbon dioxide are under development, especially those based on the physical adsorption. This study integrates this class of processes and used a biochar produced through the pyrolysis of mangaba seeds (Hancornia speciosa) as adsorbent material. Biochar was characterized in terms of surface area, pore diameter, morphology and determination of functional groups. The adsorption of $\mathrm{CO}_{2}$ was performed using the static volumetric technique, by varying the initial pressure of the system, which consisted of a fixed-bed column on a semipilot scale. The experimental data were adjusted to models in the literature. The Freundlich model was the best fit, thus confirming the adsorption in multilayers. The adsorption kinetics followed the intraparticle diffusion model, indicating that diffusion is the limiting step of the process. The higher adsorption capacity was verified, with a maximum value of $5.21 \mathrm{~mol} \mathrm{CO} 2 / \mathrm{kg}$ of bioadsorbent.

Keywords: Global warming; adsorption; biochar.

\section{INTRODUÇÃO}

O efeito estufa é um fenômeno natural e de extrema importância para o equilíbrio da vida em nosso planeta. Os gases presentes na atmosfera terrestre, também conhecidos como gases de efeito estufa (GEEs), retém o calor irradiado pela superfície da Terra e, com isso, mantém o globo aquecido e propenso à vida [1]. Porém, as ações antrópicas têm causado um aumento na concentração dos GEEs, incluindo principalmente o vapor de água $\left(\mathrm{H}_{2} \mathrm{O}\right)$, o dióxido de carbono $\left(\mathrm{CO}_{2}\right)$, o metano $\left(\mathrm{CH}_{4}\right)$, ozônio $\left(\mathrm{O}_{3}\right)$, óxidos de nitrogênio $\left(\mathrm{NO}_{\mathrm{x}}\right)$ e hidrofluorcabonetos (HFCs) [1]. 
Apontados pelos cientistas como os principais responsáveis pela intensificação do aquecimento global, os poluentes gerados em grandes quantidades pela indústria e em outros setores econômicos, são lançados diretamente na atmosfera sem tratamento, o que leva a sérias consequências tanto para o clima quanto para a saúde humana, os ecossistemas, a agricultura e o meio ambiente. Em 2014, o $\mathrm{CO}_{2}$ contribuiu com $77,7 \%$ do total de emissões, seguido do $\mathrm{CH}_{4}$, com parcela de $14,9 \%$, do $\mathrm{N}_{2} \mathrm{O}$, com $5,1 \%$, e outros gases contribuintes, com 2,5\% das emissões totais [2].

De acordo com o Painel Intergovernamental sobre Mudanças Climáticas (2014), apesar do grande número de políticas de mitigação de emissão de gases de estufa, essas emissões aumentaram significativamente nos últimos anos. No período anterior à Revolução Industrial, o nível atmosférico de dióxido de carbono correspondia a 280 ppm; em 2013, sua concentração foi de 400 ppm, o que correspondeu a um aumento da temperatura global em aproximadamente $0,8^{\circ} \mathrm{C}$ [3]. Para se atingir a meta de limitação do aumento da temperatura média global para abaixo de $2^{\circ} \mathrm{C}$, tornam-se necessários desafios econômicos, tecnológicos e institucionais [4]. Existem tecnologias avançadas que podem ser aplicadas para sequestrar o $\mathrm{CO}_{2}$ da corrente do gás antes de ser enviado para o transporte, como, por exemplo, absorção, adsorção, separação por membrana, destilação criogênica, dentre outros [5], porém a que tem se mostrado mais eficiente e mais viável economicamente é o processo de adsorção física.

A adsorção pode ser descrita de forma simplificada como sendo a adesão de moléculas a uma superfície. O termo adsorbato se refere às moléculas da fase líquida ou gasosa capturadas, e o termo adsorvente se refere ao sólido que retém o adsorbato [6]. Durante o processo de adsorção existe uma diminuição na energia livre do sistema, sendo o processo espontâneo $(\Delta \mathrm{G}<0)$. Como $\Delta \mathrm{G}=\Delta \mathrm{H}-\mathrm{T} \Delta \mathrm{S}$, a variação de entalpia geralmente será negativa, implicando que a adsorção é um processo normalmente exotérmico [7]. Esse processo é utilizado em diversas aplicações, a exemplo da recuperação de um produto valioso, da separação de odores e impurezas de gases industriais e até mesmo em aplicações caseiras em ventilação exaustora. Dentre os benefícios de se utilizar o processo de adsorção, encontra-se a durabilidade do adsorvente; a seletividade do adsorvente; a capacidade adsortiva e a estabilidade do adsorvente após muitos ciclos de adsorção/dessorção [8].

A captura do dióxido de carbono por processos de adsorção é principalmente baseada na preferência de adsorção desse gás em um meio poroso. A primeira e mais importante etapa consiste em encontrar um adsorvente adequado. Os materiais mais convencionais são as zeólitas naturais, biochars, carvões ativados e materiais baseados em aminas e sítios com basicidade de Lewis. $\mathrm{O}$ processo de adsorção física de $\mathrm{CO}_{2}$ em diversos adsorventes tem despertado o interesse científico pelo conhecimento dos mecanismos e das interações intermoleculares que justificam o sequestro do dióxido de carbono na superfície dos adsorventes [9].

Diante deste contexto, o presente trabalho teve como objetivo avaliar a capacidade adsortiva do biochar produzido a partir de sementes de mangaba (Hancornia speciosa) frente ao dióxido de carbono, numa coluna de adsorção de leito fixo, visando à captura de $\mathrm{CO}_{2}$.

\section{MATERIAL E MÉTODOS}

O adsorvente escolhido foi um biochar - material recentemente patenteado para uso na remoção de óleos e graxas [10]. É um material de origem vegetal que foi produzido a partir de uma fruta de grande ocorrência no Norte e Nordeste do Brasil: a mangaba. Através da pirólise dos caroços de seus frutos, resultou em um tipo de carvão vegetal atualmente mais conhecido como biochar. A adsorção foi estudada através de isotermas, que relaciona a quantidade de adsorbato retida pelo adsorvente com a pressão de equilíbrio de adsorção (P). A temperatura (T) e os demais parâmetros mantidos constantes.

\section{Caracterização do adsorvente}

O adsorvente foi caracterizado de acordo com sua área superficial, diâmetro dos poros, morfologia e determinação dos grupos funcionais. Para o cálculo da área superficial e do diâmetro dos poros foi usada a técnica baseada na adsorção de $\mathrm{N}_{2}$ a $77 \mathrm{~K}$, tendo sido utilizado um 
equipamento de marca NOVA®, modelo 1200e. Para realizar a análise por microscopia eletrônica de varredura (MEV), o microscópio eletrônico de varredura utilizado foi da marca JEOL $®$, modelo JSM-5700, a voltagem utilizada no experimento foi de $5 \mathrm{Kv}$ e a resolução com uma aproximação de 500 vezes. Para a análise espectroscópica por infravermelho com transformada de Fourier (FTIR), foi utilizada a técnica da pastilha de $\mathrm{KBr}$, o espectrômetro utilizado para realizar essa análise foi da marca SHIMADZUß, modelo IRPrestige-21.

\section{Obtenção das isotermas}

A técnica utilizada para obtenção das isotermas foi a volumétrica estática, na qual uma quantidade conhecida de gás foi admitida ao recipiente contendo a massa pré-determinada do adsorvente, mantendo-se a temperatura constante. A quantidade de gás adsorvido é medida conforme a adsorção ocorre, com diminuição da pressão no volume confinado até que o equilíbrio seja estabelecido [11].

O equipamento utilizado para obtenção dos dados encontra-se ilustrado na Figura 1.

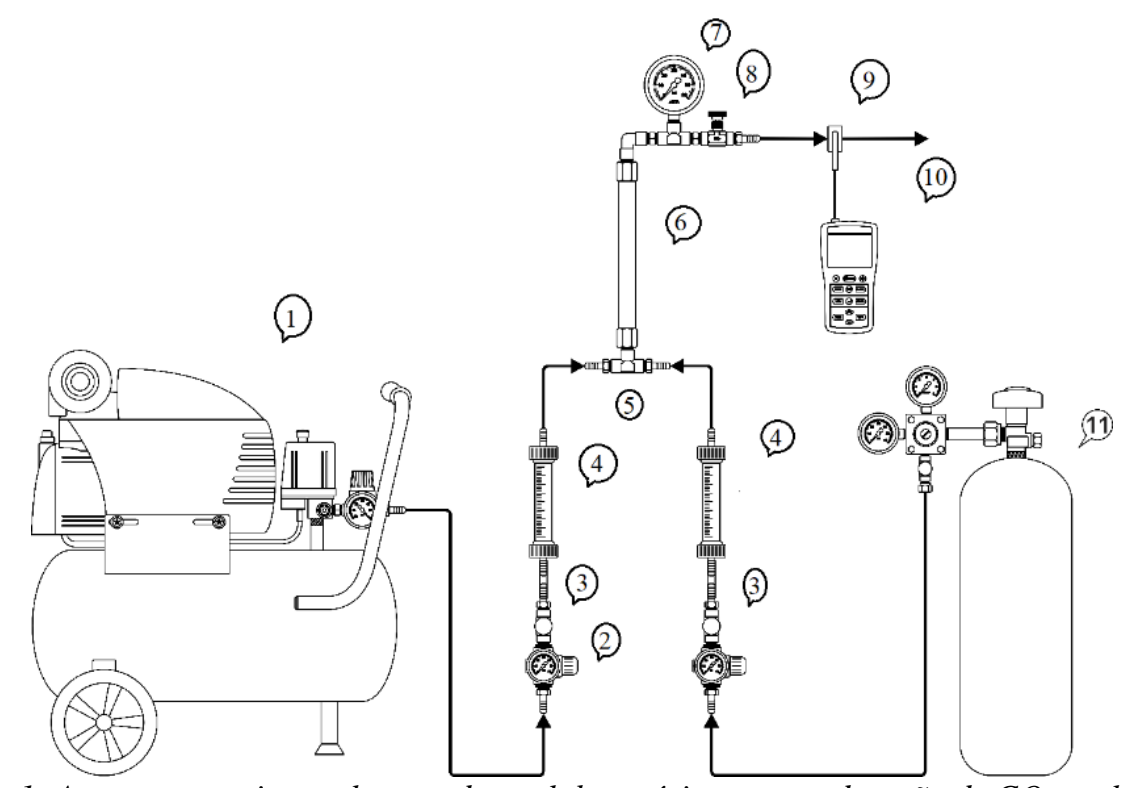

Figura 1- Aparato experimental montado em laboratório para a adsorção de $\mathrm{CO}_{2}$ em leito fixo

Onde:

1. Compressor de ar;

2. Válvulas reguladoras de pressão que visam manter a pressão de alimentação constante;

3. Válvulas de agulha em latão, cuja finalidade é controlar a vazão do gás;

4. Rotâmetros marca CONAUT ${ }^{\circledR}$ para medição de vazões de gases na faixa de 0 a 10 NL.min ${ }^{-1}$, calibrado para ar atmosférico.

5. Válvula de agulha em latão;

6. Dimensões: $52,5 \mathrm{~cm}$ de comprimento, $52 \mathrm{~mm}$ de diâmetro externo e $42 \mathrm{~mm}$ de diâmetro interno. O volume total da coluna é de $172 \mathrm{~cm}^{3}$;

7. Um manômetro tipo bourdon, com faixa de pressões de 0 a 10 bar;

8. Válvula de agulha;

9. Um canister em acrílico no qual será inserido o sensor para medição da concentração de dióxido de carbono no gás que sai da coluna;

10. Medidor da concentração de $\mathrm{CO}_{2}$, marca Instrutherm®, modelo $\mathrm{CO}_{2}$ Analyzer.

11. Cilindro contendo dióxido de carbono da White Martins ${ }^{\circledR}, 99,5 \%$ de $\mathrm{CO}_{2}$.

A quantidade de gás adsorvida à pressão de equilíbrio foi calculada pela diferença entre a quantidade de gás admitida e a quantidade de gás necessária para preencher o espaço em torno do adsorvente, isto é, o espaço morto, à pressão de equilíbrio. A isoterma de adsorção é a curva obtida 
na representação gráfica dos pontos obtidos em diversas situações experimentais, admitindo-se cargas sucessivas de gás, medindo-se a pressão de equilíbrio e determinando-se as massas adsorvidas mediante o uso de uma equação de estado. Para o presente trabalho, foi escolhida a equação de Peng-Robinson.

Os dados obtidos experimentalmente foram ajustados a modelos disponíveis na literatura, destacando-se as isotermas apresentadas na Tabela 1, analisando-se a qualidade dos ajustes por meio dos parâmetros estatísticos adequados. Os ajustes desses modelos aos dados experimentais foram realizados com o auxílio do software Statistica 6.4. O método de estimação utilizado foi o de Gauss-Newton.

Tabela 1: Isotermas de adsorção no equilíbrio [12]

\begin{tabular}{llll}
\hline Isotermas & Não-Linear & Linear & Gráfico \\
\hline Langmuir & $\mathrm{q}_{\mathrm{e}}=\mathrm{q}_{\mathrm{m}} \mathrm{K}_{\mathrm{L}} \frac{\mathrm{P}}{1+\mathrm{K}_{\mathrm{L}} \mathrm{P}}$ & $\frac{\mathrm{P}}{\mathrm{q}_{\mathrm{e}}}=\frac{1}{\mathrm{q}_{\mathrm{m}} \mathrm{K}_{\mathrm{L}}}+\frac{\mathrm{P}}{\mathrm{q}_{\mathrm{m}}}$ & $\frac{\mathrm{P}}{\mathrm{q}_{\mathrm{e}}}$ vs $\mathrm{P}$ \\
Freundlich & $\mathrm{q}_{\mathrm{e}}=\mathrm{K}_{\mathrm{f}} \mathrm{P}^{1 / \mathrm{n}}$ & $\ln \mathrm{q}_{\mathrm{e}}=\ln \mathrm{K}_{\mathrm{f}}+\frac{1}{\mathrm{n}} \ln \mathrm{P}$ & $\ln (\mathrm{qe})$ vs $\operatorname{lnP}$ \\
Toth & $\mathrm{qe}=\frac{\mathrm{K}_{\mathrm{T}} \mathrm{P}}{\left(\alpha_{\mathrm{T}}+\mathrm{P}\right)^{1 / \mathrm{t}}}$ & $\ln \left(\frac{\mathrm{q}_{\mathrm{e}}}{\mathrm{K}_{\mathrm{T}}}\right)=\ln \mathrm{P}-\frac{1}{\mathrm{t}} \ln \left(\alpha_{\mathrm{T}}+\mathrm{P}\right)$ & $\ln \left(\frac{\mathrm{q}_{\mathrm{e}}}{\mathrm{K}_{\mathrm{T}}}\right)$ vs $\operatorname{lnP}$ \\
\hline
\end{tabular}

Onde P é a pressão do sistema, em bar; $\mathrm{q}_{\mathrm{e}}$ é a concentração do adsorbato na fase sólida em

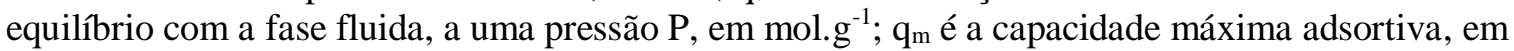
mol. $\mathrm{g}^{-1} ; \mathrm{K}_{\mathrm{L}}$ é a constante de Langmuir relacionada à energia de ligação entre o adsorbato $\mathrm{e} \mathrm{o}$ adsorvente $\left(\mathrm{g} \cdot \mathrm{mol}^{-1}\right) ; \mathrm{K}_{\mathrm{f}}$ é o coeficiente de adsorção de Freundlich, relacionado à capacidade adsortiva (mol.g $\left.{ }^{-1}\right) ; \mathrm{n}$ é um parâmetro empírico que se relaciona com a intensidade de adsorção; $\alpha_{\mathrm{T}}$ e t são constantes da isoterma de Toth.

\section{Estudo da cinética de adsorção}

A cinética de adsorção foi investigada para o biochar. A temperatura do sistema foi a ambiente $(25 \pm 1)^{\circ} \mathrm{C}$ e o sistema encontrava-se pressurizado a 2 bar. Em intervalos de tempo prédeterminados, a queda de pressão do sistema foi registrada e a sua respectiva capacidade adsortiva foi calculada.

Foram analisados os ajustes dos modelos de pseudo-primeira ordem, pseudo-segunda ordem e difusão intrapartícula (Tabela 2), comparando-se os coeficientes de determinação $\left(\mathrm{R}^{2}\right)$ e a função do erro médio relativo (ARE).

Tabela 2- Modelos cinéticos

\begin{tabular}{ll}
\hline Modelos cinéticos & Equação \\
\hline Pseudo-primeira ordem & $\frac{d q_{t}}{d t}=k 1\left(q_{e}-q_{t}\right)$ \\
Pseudo-segunda ordem & $\frac{d q_{t}}{d t}=k 2\left(q_{e}-q_{t}\right)^{2}$ \\
Difusão intrapartícula & $q_{t}=K_{d i f} t^{0,5}+C$ \\
\hline
\end{tabular}

\section{RESULTADOS E DISCUSSÃO}

A área superficial do biochar (Tabela 3) foi obtida através da técnica de BET (Brunauer, Emmet e Teller). Percebe-se que a área superficial do biochar foi relativamente alta quando comparada com outros dados da literatura (Tabela 4), concluindo-se, portanto, que não há a necessidade de funcionalização do material. Sua área superficial é semelhante à área superficial do biochar produzido a partir da pirólise da madeira e da espiga de milho (Tabela 4). 
Tabela 3- Caracterização textural do sólido adsorvente.

\begin{tabular}{l|l}
\hline Propriedades & Biochar \\
\hline Volume total dos poros, $\mathbf{c m}^{\mathbf{3}} \cdot \mathbf{g}^{-1}$ & 0,11 \\
Área superficial, $\mathbf{~ m}^{\mathbf{2}} \cdot \mathbf{g}^{-1}$ & 137 \\
Diâmetro médio do poro, $\AA$ & 16 \\
Diâmetro médio da partícula, $\mathbf{m m}$ & 0,56 \\
Massa específica real, $\mathbf{g . c m}$ & $\mathbf{- 3}$ \\
\hline
\end{tabular}

Interpretando a caracterização textural (Tabela 3), destaca-se que o material pode ser classificado como microporoso, por possuir um diâmetro médio do poro menor que $20 \AA$. Baseado nas propriedades dos poros, a temperatura de pirólise de $600^{\circ} \mathrm{C}$ foi adequada à produção do biochar, uma vez que, uma elevada área superficial está conectada a uma boa porosidade e, por consequência, ao aumento da capacidade adsortiva [13].

Tabela 4- Área superficial e temperatura de pirólise de biochars disponíveis na literatura.

\begin{tabular}{l|ccc}
\hline Origem do biochar & $\begin{array}{c}\text { Temperatura } \\
\text { de pirólise, }{ }^{\circ} \mathrm{C}\end{array}$ & Área superficial, $\mathbf{m}^{\mathbf{2}} \mathbf{g}^{-\mathbf{1}}$ & Referência \\
\hline $\begin{array}{l}\text { Lodo de digestão anaeróbia } \\
\text { Torta de mamona }\end{array}$ & 800 & 101 & {$[14]$} \\
Torta de mamona & 400 & 4 & {$[15]$} \\
Espiga de milho & 550 & 3 & {$[13]$} \\
Madeira & 600 & 123 & {$[16]$} \\
Casca de arroz & 600 & 147 & {$[16]$} \\
Serragem & 600 & 11 & {$[16]$} \\
Palha de trigo & 600 & 7 & {$[16]$} \\
Sementes de mangaba & 700 & 26 & {$[17]$} \\
\hline
\end{tabular}

A determinação dos grupos funcionais do biochar foi realizada através da técnica de FT-IR (Espectroscopia de Infravermelho com Transformada de Fourier). Ampliando-se o espectro nas bandas entre 2000 e $1000 \mathrm{~cm}^{-1}$ (Figura 3), constatam-se vibrações aproximadamente perto da banda $1700 \mathrm{~cm}^{-1}$, que estão provavelmente relacionadas a ligações $\mathrm{C}=\mathrm{O}$ em ácidos carboxílicos [15]. Os estiramentos vibracionais observados entre as bandas 1600 e $1400 \mathrm{~cm}^{-1}$ são provavelmente relacionadas às ligações $\mathrm{C}=\mathrm{C}$ e $\mathrm{C}-\mathrm{C}[18]$.

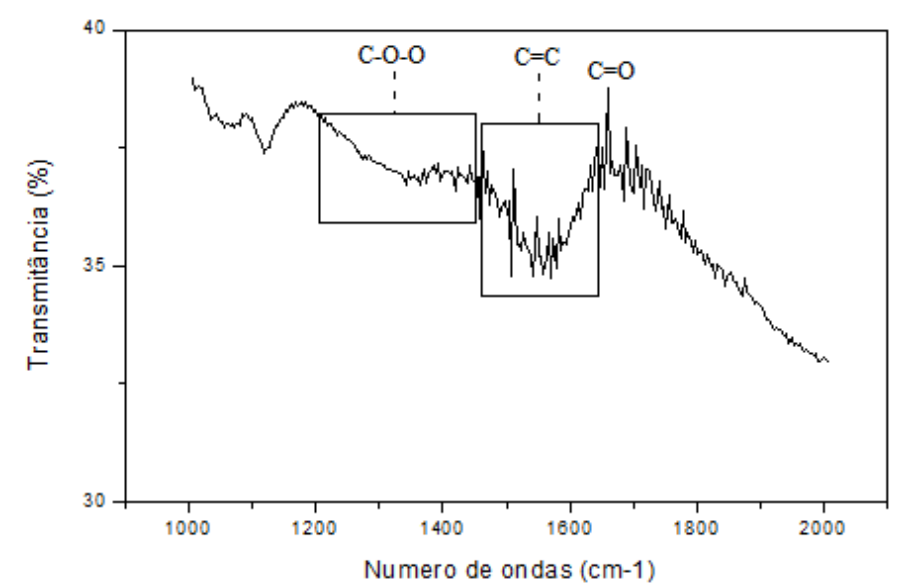

Figura 2- Espectro de FT-IR para o biochar, com aproximação entre as bandas $2000 \mathrm{~cm}^{-1} \mathrm{e} 1000 \mathrm{~cm}^{-1}$.

A caracterização morfológica do material foi realizada através do microscópio eletrônico de varredura. Boa parte da porosidade que pode ser vista na análise morfológica do biochar (Figura 4) é resultado da resistência das estruturas dos materiais, como por exemplo as paredes celulares e 
os vasos condutores. A alta temperatura $\left(600^{\circ} \mathrm{C}\right)$ de pirólise a qual o material foi submetido, proporciona a liberação de material volátil, contribuindo para a formação dos poros [19].

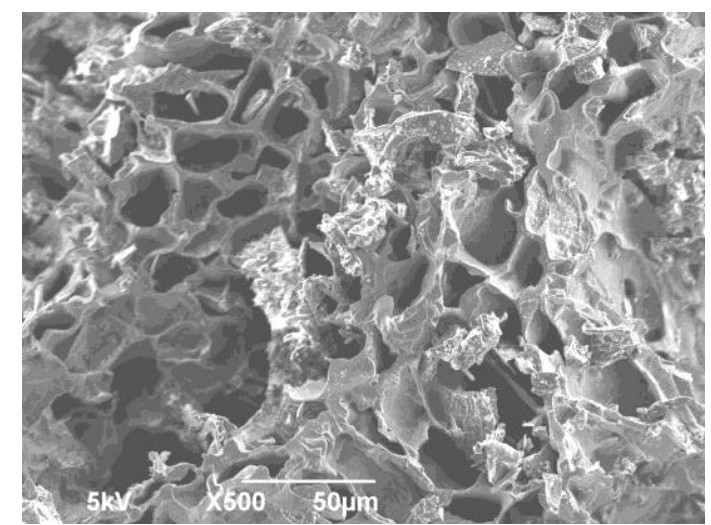

Figura 3- Microscopia eletrônica de varredura do biochar com ampliação de 500 vezes.

As isotermas de equilíbrio de adsorção de $\mathrm{CO}_{2}$ sobre o biochar foram feitas no Laboratório de Controle da Poluição (LCPA) no Departamento de Engenharia Ambiental da Universidade Federal de Sergipe. $\mathrm{O}$ adsorvente foi previamente regenerado a uma temperatura de $180^{\circ} \mathrm{C}$ e um tempo de 1 hora com o objetivo de remover a presença da umidade.

Os resultados dos ensaios volumétricos estáticos realizados na coluna de adsorção desenvolvida para o presente estudo com uso do biochar, foram convertidos em gráficos relacionando a capacidade de adsorção com a pressão de equilíbrio, para cada pressão inicial (ou massa de $\mathrm{CO}_{2}$ inicial). Os experimentos estão descritos na Tabela 5.

Tabela 5-Resultados da capacidade máxima adsortiva para o biochar.

\begin{tabular}{c|ccc}
\hline Experimento & Pressão inicial (bar) & Temperatura $\left({ }^{\circ} \mathrm{C}\right)$ & $\begin{array}{c}\text { Capacidade } \\
\text { de adsorção }\left(\mathbf{m o l . k g}^{-\mathbf{1}}\right)\end{array}$ \\
\hline $\mathbf{1}$ & 2,0 & 25 & 1,59 \\
$\mathbf{2}$ & 2,6 & 25 & 2,40 \\
$\mathbf{3}$ & 3,0 & 25 & 3,21 \\
$\mathbf{4}$ & 3,6 & 25 & 3,78 \\
$\mathbf{5}$ & 4,0 & 25 & 4,83 \\
$\mathbf{6}$ & 4,6 & 25 & 5,21 \\
\hline
\end{tabular}

Na Figura 5 ilustra-se a isoterma de adsorção para o biochar na faixa de pressões de 2 a 4,6 bar. A partir da pressão inicial de 2 bar, a capacidade de adsorção cresceu de modo intenso e aproximadamente linear até a pressão inicial 4,6 bar, com tendência à saturação em pressões mais elevadas. Pode-se observar que a capacidade de adsorção aumenta com o aumento das pressões de equilíbrios na temperatura de $25^{\circ} \mathrm{C}$. Esse fator pode ser atribuído à natureza exotérmica do processo de adsorção [20].

A tabela 6 mostra a comparação entre a capacidade adsortiva do biochar com outros materiais adsorventes frente ao sequestro de $\mathrm{CO}_{2}$. A partir desse estudo comparativo, é possível observar que o biochar de sementes de mangaba tem uma capacidade adsortiva consideravelmente maior que os materiais que serviram como base de comparação para esse estudo. 


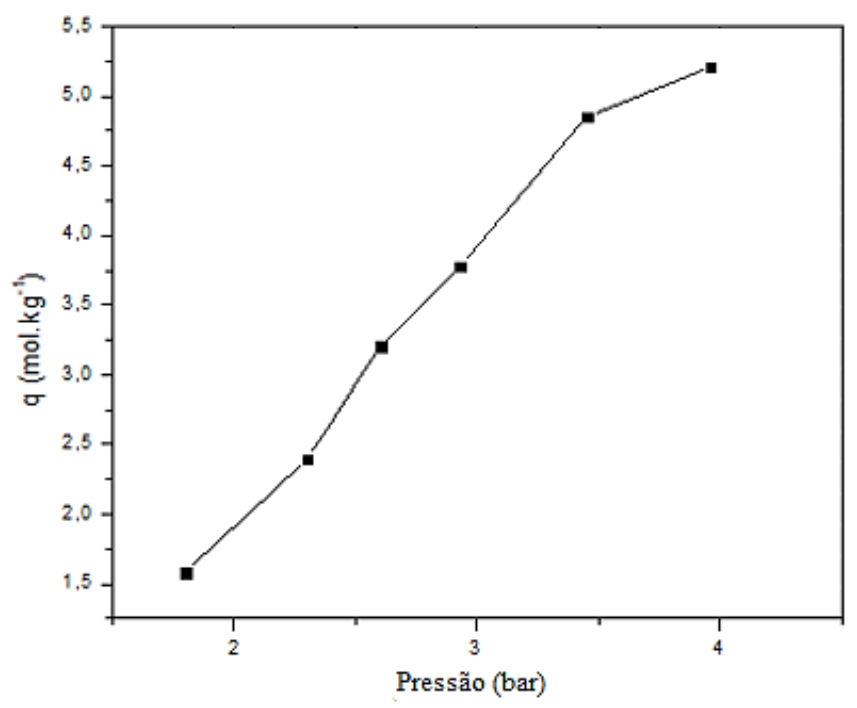

Figura 4- Isoterma de adsorção do $\mathrm{CO}_{2}$ no biochar para a temperatura do experimento de $25^{\circ} \mathrm{C}$.

Tabela 6- Capacidade adsortiva máxima de adsorventes disponíveis na literatura

\begin{tabular}{l|cccc}
\hline \multicolumn{1}{l}{ Biochar } & Adsorvato & $\begin{array}{l}\text { Temperatura de } \\
\text { adsorção }\left({ }^{\circ} \mathrm{C}\right)\end{array}$ & $\begin{array}{l}\text { Capacidade } \\
\text { de adsorção } \\
\left(\mathbf{m o l . k g} \mathbf{1}^{-1}\right)\end{array}$ & Referência \\
\hline $\begin{array}{l}\text { Casca de amêndoa } \\
\text { Serragem }\end{array}$ & $\mathrm{CO}_{2}$ & 25 & 2,7 & {$[21]$} \\
Bambu & $\mathrm{CO}_{2}$ & 25 & 3,2 & {$[22]$} \\
Caule de algodão & $\mathrm{CO}_{2}$ & 25 & 4,5 & {$[23]$} \\
$\begin{array}{l}\text { Madeira } \\
\text { Caroço de azeitona }\end{array}$ & $\mathrm{CO}_{2}$ & 20 & 2,18 & {$[24]$} \\
$\begin{array}{l}\text { Biochar de sementes de } \\
\text { mangaba }\end{array}$ & $\mathrm{CO}_{2}$ & 25 & 1,11 & {$[25]$} \\
\hline
\end{tabular}

Os dados experimentais foram ajustados aos modelos de isotermas de Langmuir, Freundlich e Toth e os parâmetros dos ajustes aos modelos e os coeficientes de determinação podem ser vistos na Tabela 7. A indicação da favorabilidade da adsorção pode ser feita através do fator de separação de Langmuir, $R_{L}$. Quando $R_{L}>1$ a isoterma não é favorável, quando $R_{L}<1$ a isoterma é favorável e quando $R_{L}=0$, irreversível. $O$ valor do $R_{L}$ calculado ficou dentro da faixa de 0 a 1 , o que sugere que a isoterma seja favorável [26].

Levando em consideração os valores dos coeficientes de determinação $\left(\mathrm{R}^{2}\right)$, o modelo de Freundlich (Figura 6) apresenta o melhor ajuste aos dados experimentais, com um $\mathrm{R}^{2}$ de 0,9658 . Esse modelo indica que as moléculas adsorvidas formam camadas sucessivas na superfície do adsorvente, implicando que é provável que a adsorção tenha ocorrido em multicamadas nos poros do biochar à temperatura de $25^{\circ} \mathrm{C}$ [26]. 


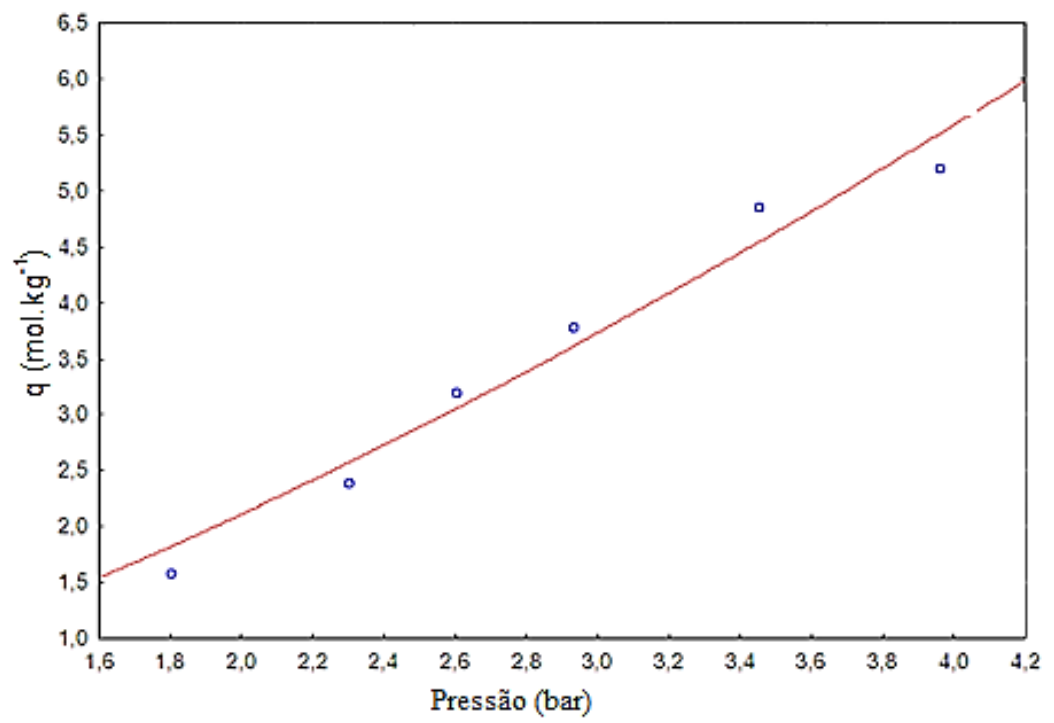

Figura 5- Isoterma de adsorção do $\mathrm{CO}_{2}$ no biochar ajustada ao modelo de Freundlich.

Tabela 7-Modelos de isotermas e seus respectivos parâmetros e coeficientes de determinação.

\begin{tabular}{l|ll}
\hline Isotermas & Parâmetros & $\mathbf{R}^{2}$ \\
\hline Langmuir & $\mathrm{qm}=186,7161\left(\mathrm{~mol}_{\mathrm{g}} \mathrm{g}^{-1}\right)$ & 0,9413 \\
Freundlich & $K_{L}=0,0067\left(\mathrm{~g} \cdot \mathrm{mol}^{-1}\right)$ & \\
$\mathrm{k}=0,7971\left(\mathrm{~mol}_{\mathrm{g}} \mathrm{g}^{-1}\right)$ & 0,9832 \\
Toth & $\mathrm{n}=0,7120$ & \\
& $\mathrm{k}=1,2888\left(\mathrm{~mol} \cdot \mathrm{g}^{-1}\right)$ & 0,9447 \\
& $\alpha=530,68$ & \\
$\mathrm{t}=0,0019$ & \\
\hline
\end{tabular}

O estudo cinético foi realizado a uma temperatura de $25^{\circ} \mathrm{C}$, pressão de 2 bar e ajustado aos três modelos cinéticos escolhidos (pseudo-primeira ordem, pseudo-segunda ordem e difusão intrapartícula) para melhor entender o processo que governa a adsorção do adsorvente estudado. Os dados cinéticos experimentais mostram que o equilíbrio foi atingido em menos de 250 minutos, com um suave aumento da capacidade adsortiva a partir de 200 minutos (Figura 7).

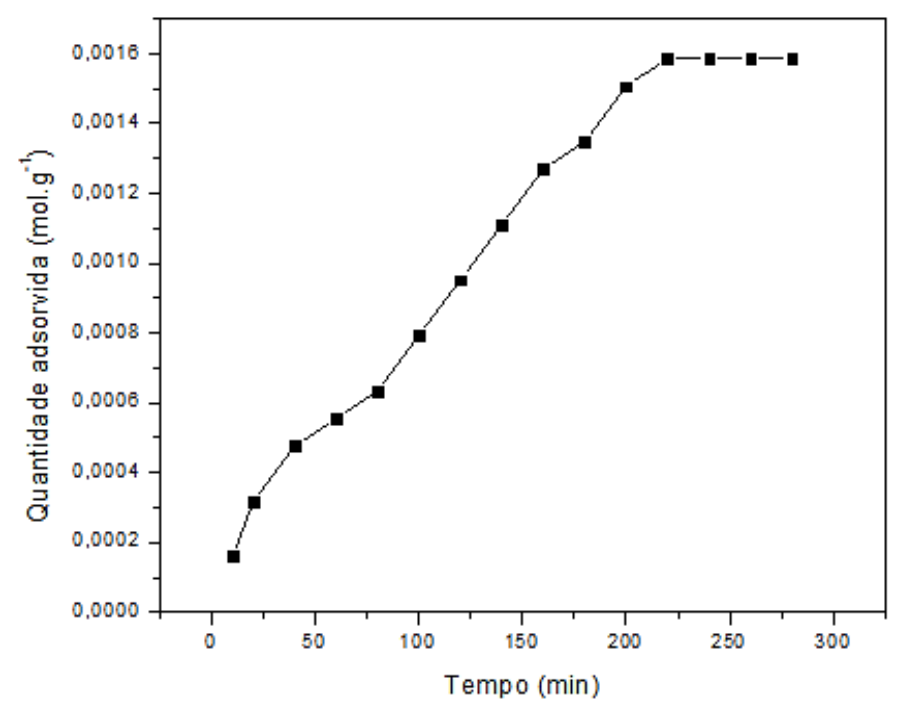

Figura 6-Cinética de adsorção do biochar; temperatura de $25^{\circ} \mathrm{C}$ e pressão de 2 bar. 
Os valores dos parâmetros cinéticos para cada modelo e seus respectivos coeficientes de determinação e porcentagens de erro encontram-se na Tabela 8. Através da análise quantitativa dos coeficientes de determinação e das porcentagens de erro, conclui-se que o modelo de difusão intrapartícula proporciona o melhor ajuste aos dados experimentais.

Os dados obtidos pela técnica de BET e pela microscopia eletrônica de varredura comprovam que o biochar possui uma grande área superficial e uma estrutura interna desenvolvida, justificando, portanto, a hipótese de ocorrência da difusão intrapartícula.

Baseado no bom ajuste ao modelo de difusão intrapartícula, conclui-se que o processo de adsorção aconteceu em três etapas: adsorção na superfície, seguida da adsorção gradual, na qual a difusão intrapartícula é etapa limitante e, por último, ocorre a fase do equilíbrio, quando a difusão intrapartícula provoca a redução da velocidade devido à menor quantidade de sítios de adsorção acessíveis.

Tabela 8- Modelos cinéticos com seus respectivos parâmetros, coeficientes de determinação e porcentagens de erro

\begin{tabular}{l|llr}
\hline \multicolumn{1}{c}{ Modelos cinéticos } & \multicolumn{1}{c}{ Parâmetros cinéticos } & \multicolumn{1}{c}{$\mathbf{R}^{2}$} & ARE (\%) \\
\hline Pseudo-primeira ordem & $q_{e}=0,002245\left(\mathrm{~mol}_{\mathrm{g}} \mathrm{g}^{-1}\right)$ & 0,9900 & 0,4549 \\
& $\mathrm{k}=0,004952(\mathrm{~min})^{-1}$ & & 0,3340 \\
Pseudo-segunda ordem & $\mathrm{q}=0,003527\left(\mathrm{~mol} \cdot \mathrm{g}^{-1}\right)$ & 0,9895 & \\
& $\mathrm{k}=0,938131\left(\mathrm{~mol} \cdot \mathrm{g}^{-1} \mathrm{~min}^{-1}\right)$ & & 0,2816 \\
Difusão intrapartícula & $\begin{array}{l}\mathrm{K}_{\mathrm{dif}}=0,000118\left(\mathrm{~mol} \cdot \mathrm{g}^{-1} \mathrm{~min}^{-0,5}\right) \\
\mathrm{C}=-0,000282\left(\mathrm{~mol} \cdot \mathrm{g}^{-1}\right)\end{array}$ & 0,9865 & \\
\hline
\end{tabular}

\section{CONCLUSÃO}

O biochar produzido a partir da semente de mangaba (Hancornia Speciosa) exibe propriedades que corroboram sua adequação como excelente material adsorvente para $\mathrm{CO}_{2}$. Os dados experimentais foram ajustados a três tipos diferentes de isotermas: Langmuir, Freundlich e Toth, sendo que a que melhor se ajustou foi a isoterma de Freundlich, reforçando a hipótese de adsorção em multicamadas.

Os experimentos de adsorção do dióxido de carbono no biochar mostraram que o mesmo tem uma elevada capacidade adsortiva, atingindo cerca de $5,21 \mathrm{~mol} \cdot \mathrm{kg}^{-1}$, o que equivale a $229,24 \mathrm{mg}$ de $\mathrm{CO}_{2} \cdot \mathrm{g}^{-1}$ de carvão, a $25^{\circ} \mathrm{C}$, para uma pressão inicial de aproximadamente 4,6 bar. A capacidade máxima adsortiva do biochar mostrou-se coerente com os resultados encontrados na literatura. $\mathrm{O}$ estudo cinético classificou a difusão intrapartícula como a etapa limitante do processo.

A alta capacidade adsortiva sugere que o biochar produzido a partir das sementes de mangaba é um adsorvente promissor para a captura de $\mathrm{CO}_{2}$.

\section{AGRADECIMENTOS}

A CAPES (Coordenação de Aperfeiçoamento de Pessoal de Nível Superior) pelo fomento disponibilizado à esta pesquisa.

\section{REFERÊNCIAS BIBLIOGRÁFICAS}

1. Prasad PVV, Thomas JMG, Narayanan S. Global Warming Effects. Encyclopedia of Applied Plant Sciences. 2017 Sep;3:289-299, doi:10.1016/B978-0-12-394807-6.00013-7.

2. National Greenhouse Gas Inventory Data for the period 1990-2014. United Nations. Framework Convention on Climate Change, UNFCCC; 2016.

3. Intergovernmental Panel on Climate Change- IPCC; 2014.

4. Conferência sobre Mudanças Climáticas das Nações Unidas - COP 21, Paris; 2015. 
5. Leung DYC, Caramanna G.; Maroto-Valer MM. An overview of current status of carbon dioxide capture and storage technologies. Renewable and Sustainable Energy Reviews. 2014 Nov;39:426-443, doi:10.1016/j.rser.2014.07.093.

6. Mansour RB, Habib MA, Bamidele OE, Basha M, Qasem NAA, Peedikakkal A, Laoui T, Ali M. Carbon capture by physical adsorption: materials, experimental investigations and numerical modelling and simulation- a review. Applied Energy, 2016 Jan;161:225-255, doi:10.1016/j.apenergy.2015.10.011.

7. Ruthven DM. Principles of adsorption and adsorption processes. John Wiley and Sons. 1984;467-524.

8. Songolzadeh M, Ravanchi MT, Soleimani M. Carbon dioxide capture and storage: a general review on adsorvents. World Academy of Science, Engineering and Technology. 2012;6:10.

9. Dias MFC. Estudos De Adsorção De Co2 Gasoso Em Biocarvão (Biochar) [Dissertation]. Coimbra (PT): Universidade de Coimbra; 2014.

10. Leite NS, Silva RR, Marques JJ, Tambourgi EB, Silva GF, Freitas LS, Santos RM, inventores; Universidade Federal de Sergipe, depositante. Uso de um bioadsorvente produzido a partir de sementes de mangaba para a remoção de contaminantes de águas e efluentes líquidos. BR 102017001445 2. 2017 Jan 24.

11. Sing KSW. Reporting physisorption data for gas/solid systems with special reference to the determination of surface area and porosity. Pure \& Applied Chemistry. 1982;54:2201-2218.

12. Dehghani MH, Dehghan A, Najafpoor A. Removing Reactive Red 120 and 196 using chitosan/zeolite composite from aqueous solutions: Kinetics, isotherms, and process optimization. Journal of Industrial and Engineering Chemistry. 2017 Mar;51:185-195, doi:10.1016/j.jiec.2017.03.001.

13. Hilioti Z, Michailof CM, Valasiadis D, Iliopoulou EF, Koidou V, Lappas AA. Characterization of castor plant-derived biochar and their effects as soil amendments on seedlings. Biomass and Bioenergy. 2017 Oct;105:96-106, doi:10.1016/j.biombioe.2017.06.022.

14. Hung CH, Tsai WT, Chen JW, Lin YQ, Chang, YM. Characterization of biochar prepared from biogas digestate. Waste Management. 2017 Aug;66:53-60, doi:10.1016/j.wasman.2017.04.034.

15. Kalinke C, Oliveira PR, Oliveira GA, Mangrich AS, Junior LH, Bergamini MF. Activated biochar: Preparation, characterization and electroanalytical application in an alternative strategy of nickel determination, Analytica Chimica Acta. 2017 Aug;983:103-111, doi:10.1016/j.aca.2017.06.025.

16. Kizito S, Luo H, Wu S, Ajmal Z, Lv T, Dong R. Phosphate recovery from liquid fraction of anaerobic digestate using four slow pyrolyzed biochars: Dynamics of adsorption, desorption and regeneration. Journal of Environmental Management. 2017 Oct;201:260-267, doi:10.1016/j.jenvman.2017.06.057.

17. Oleszczuk P, Bundyra WC, Bogusz A, Skwarek E, Ok YS. Characterization of nanoparticles of biochars from different biomass. Journal of Analytical and Applied Pyrolysis. 2016 Sep;121:165-172, doi:10.1016/j.jaap.2016.07.017.

18. Luo L, Xu C, Chen Z, Zhang S. Properties of biomass-derived biochars: Combined effects of operating conditions and biomass types. Bioresource Technology. 2015 Sep;192:83-89, doi:10.1016/j.biortech.2015.05.054.

19. Taherymoosavi S, Verheyen V, Munroe P, Joseph S, Reynolds A. Characterization of organic compounds in biochars derived from municipal solid waste. Waste Management. 2017 Sep;67:131-142, doi:10.1016/j.wasman.2017.05.052.

20. Jedli H, Jbara A, Hedfi H, Bouzgarrou S, Slimi K. Carbon dioxide adsorption isotherm on various cap rock in a batch reactor for $\mathrm{CO}_{2}$ sequestration processes. Applied Clay Science. 2017 Feb;136:199-207, doi:10.1016/j.clay.2016.11.022.

21. Plaza MG, Garcia S, Rubiera F, Pis JJ, Pevida C. Post-combustion $\mathrm{CO}_{2}$ capture with a commercial activated carbon: Comparison of different regeneration strategies. Chemical Engineering Journal. 2010 Sep;163:41-47, doi:10.1016/j.cej.2010.07.030.

22. Sevilla M, Fuertes AB. $\mathrm{CO}_{2}$ adsorption by activated templated carbons. Journal of Colloid and Interface Science. 2012 Jan;366:147-154, doi:10.1016/j.jcis.2011.09.038.

23. Wei H, Deng S, Hu B, Chen Z, Wang B, Huang J, Yu G. Granular Bamboo-Derived Activated Carbon for High CO2 Adsorption: The Dominant Role of Narrow Micropores. ChemSusChem. 2012 Nov;5:2354-2360, doi:10.1002/cssc.201200570.

24. Zhang X, Zhang S, Yang H, Feng Y, Chen Y, Wang X, Chen H. Nitrogen enriched biochar modified by high temperature $\mathrm{CO} 2$-ammonia treatment: Characterization and adsorption of $\mathrm{CO}_{2}$. Chem. Eng. J. 2014 Dec;257:20-27, doi:10.1016/j.cej.2014.07.024.

25. Plaza MG, Previda C, Arias B, Fermoso J, Casal MD, Martin CF, Rubiera F, Pis JJ. Development of lowcost biomass-based adsorbents for postcombustion $\mathrm{CO}_{2}$ capture. Fuel. 2009 Dec;88:2442-2447, doi:10.1016/j.fuel.2009.02.025

26. Rashidi NA, Yusup S, Borhan A. Isotherm and termodynamic analysis of carbon dioxide on activated carbon. Procedia Engineering. 2016;148:630-637, doi:10.1016/j.proeng.2016.06.527. 